\title{
Platelet-rich plasma-enhanced osseointegration of decellularized bone matrix in critical-size radial defects in rabbits
}

\author{
Yi Leng, Guangkai Ren, Yutao Cui, Chuangang Peng, Jincheng Wang, Dankai Wu, He Liu \\ Department of Orthopedics, The Second Hospital of Jilin University, Changchun 130041, China \\ Contributions: (I) Conception and design: Y Leng, H Liu, D Wu; (II) Administrative support: D Wu, J Wang; (III) Provision of study materials or \\ patients: G Ren, Y Cui; (IV) Collection and assembly of data: Y Leng, Y Cui; (V) Data analysis and interpretation: Y Leng, C Peng; (VI) Manuscript \\ writing: All authors; (VII) Final approval of manuscript: All authors. \\ Correspondence to: He Liu; Dankai Wu; Jincheng Wang. Department of Orthopedics, The Second Hospital of Jilin University, Changchun 130041, \\ China. Email: heliu@jlu.edu.cn; wudankai@163.com; jinchengwang@hotmail.com.
}

\begin{abstract}
Background: Bone defects represent a common orthopedic condition. With its vast array of donor sources, xenogeneic bone shows considerable potential as a bone defect repair material but may also be associated with immune rejection and reduced osteogenic capacity. Thus, reducing the risks for immune rejection of xenogeneic bone, while improving its osseointegration, are key technical challenges.

Methods: Decellularized bone matrix scaffolds (DBMs) were fabricated by thorough ultrasonic vibration and subjection to chemical biological agents to remove cells and proteins. The DBMs were then mixed with platelet-rich plasma (PRP) under negative pressure. Growth factor concentrations of PRP, as well as the microstructures and biomechanical properties of the system, were examined. Furthermore, osseointegration capacities in the critical-size radial defect rabbit model were verified.

Results: Complete decellularization of the scaffold and limited reductions in mechanical strength were observed. Moreover, the obtained PRP demonstrated various growth factors. Radiographic evaluation and histological analysis verified that more new bone formation occurred in the DBM mixed with PRP group at 6 and 12 weeks after implantation compared with both the blank group and the DBM without PRP group.

Conclusions: Thorough physical and chemical treatments can reduce the probability of immune rejection of DBMs. The novel composite of DBMs mixed with PRP can serve as a promising bone regeneration material.
\end{abstract}

Keywords: Large bone defect; xenograft; decellularization; platelet-rich plasma (PRP); bone regeneration

Submitted Oct 23, 2019. Accepted for publication Jan 02, 2020.

doi: 10.21037/atm.2020.01.53

View this article at: http://dx.doi.org/10.21037/atm.2020.01.53

\section{Introduction}

Large bone defects, caused by trauma, infection, and tumor resection, comprise one of the most troublesome problems faced by orthopedists. In some cases, the treatment of bone defects can lead to serious complications, such as nonunion, bone atrophy, and bone deformity (1-3). To date, bone grafting is considered the most effective technique in clinical practice and includes autologous bone grafting, allogeneic bone grafting, bone graft replacement, and heterogeneous bone grafting $(4,5)$.
However, autologous bone transplantation, as the clinical gold standard, is characterized by a limited supply of donors, complicated procedures, and pain at the donor site, as well as potential infection (6,7). Moreover, allograft bone is associated with several ethical issues and large costs, which can be prohibitive for some patients with large bone defects $(8,9)$. Bone graft replacements, such as inorganic bone substitutes, are usually composed of calcium phosphate and calcium sulfate processed by physical or chemical methods, but they demonstrate weak osteoinductive properties and are prone to triggering inflammatory reactions (10). 
Heterogeneous bone grafts, also called decellularized bone matrix scaffolds (DBMs), which are comprised of nonhuman cancellous bone treated by chemical and biological methods, can provide osteoconductive effects similar to those of autologous and allogeneic bone grafts (11). Moreover, the risk of disease transmission and immune rejection caused by xenotransplantation can be greatly avoided if decellularization methods are employed (12). However, after thorough processing, xenografts do not retain their natural osteoinductive factors and, thus, fail to achieve the same clinical effects as autologous bone grafts (13). Platelet-rich plasma (PRP) is defined as a plasma product containing relatively high concentrations of various growth factors, including platelet-derived growth factor (PDGF), insulin-like growth factor (IGF), and transforming growth factor- $\beta$ (TGF- $\beta$ ) (14). In addition, PRP has been widely used clinically to treat osteoarthritis and rotator cuff injuries $(15,16)$. Previous studies report that PRP could promote bone and cartilage regeneration $(17,18)$.

Based on these findings, we believe that PRP can compensate for the deficiency of porous DBMs to promote osteogenesis in critical-size bone defects. However, limited information has been reported on the combined effects of PRP and DBMs on bone healing, bone nonunion, and other outcomes. Therefore, we decided to establish a large radial defect rabbit model and observe the effects of repair using DBMs alone and the combined effects of porous DBMs with PRP filling. We hoped to provide a new solution for the treatment of bone defects.

\section{Methods}

\section{Preparation of DBMs}

The animal experiments were conducted according to the National Institutes of Health Guide for the Care and Use of Laboratory Animals and approved by Institutional Review Board (IRB) of Jilin University (IRB No. 2019132). Femurs to be used in the preparation of DBMs were obtained from freshly sacrificed female pigs, aged 3-4 years old and weighing 150-200 kg, at Huazheng Company (Changchun, Jilin, China). A new method of manufacturing DBMs was applied. In brief, methanol and chloroform solution were used as a buffer at a ratio of 1:1. The pre-shaped bone was then processed with an ultrasonic cell crusher after being soaked in the buffer for $8 \mathrm{~h}$. The initial parameters were set to $200 \mathrm{w}$, with a working frequency of $20 \mathrm{kHz}, 5 \mathrm{~s}$ for each operation with a pause of $5 \mathrm{~s}$, which was regarded as one set. A group of 300 sets was considered one cycle. After three cycles, the adipose tissue and organic solvent-soluble components in the DBMs were eliminated. The samples were then cleaned three times with phosphate-buffered saline (PBS) and double-distilled water to remove any residual tissue and buffer.

The combination of $0.5 \%$ dipase and $1 \%$ Triton-X 100 in $0.05 \mathrm{~mol} \mathrm{~L}^{-1}$ Tris-HCL buffer $(\mathrm{pH}=7.8)$ was then used to effect further denaturation of proteins and cell lysis. Samples were immersed in the buffer solution and shaken at room temperature for $8 \mathrm{~h}$, followed by placement in an ultrasonic cell breaker for $30 \mathrm{~min}$. The buffer was then replaced. This step was repeated three times. The ultrasonic parameters were set to a frequency $40 \mathrm{kHz}$ and power of $100 \mathrm{w}$. The samples were then washed three times with PBS and double-distilled water, freeze-dried, and then maintained at $-80{ }^{\circ} \mathrm{C}$ until further analysis.

\section{PRP preparation and characterization}

\section{Rabbit PRP preparation}

To prevent immune rejection, the blood of each animal was used to extract the PRP. The method of PRP preparation was adapted from that of a previous study (19). Briefly, after the skin was disinfected, approximately $4.5 \mathrm{~mL}$ of blood was obtained from the heart and mixed with $0.5 \mathrm{~mL}$ of sodium citrate solution. The blood was then centrifuged twice initially at $209 \mathrm{~g}$ for $16 \mathrm{~min}$ at $20^{\circ} \mathrm{C}$ to remove red blood cells, followed by $1,500 \mathrm{~g}$ for $12 \mathrm{~min}$ at $20^{\circ} \mathrm{C}$ to obtain the platelet pellet. The PRP was acquired by platelet pellet resuspension in platelet-poor plasma, with an average platelet number of $10.00 \times 10^{8} \mathrm{~mL}^{-1}$.

\section{Determination of growth factor content}

To determine the levels of various growth factors present in the PRP obtained, the concentrations of IGF-1, bFGF, VEGF, and PDGF were determined using an ELISA method as described previously (20).

\section{Construction of DBMs containing PRP}

\section{Construction method}

The DBMs and PRP stored at $-80^{\circ} \mathrm{C}$ were removed and placed at room temperature 1 day before preparation. In brief, the melted $300 \mu \mathrm{L}$ of PRP was evenly distributed on the surface of DBMs $(15 \mathrm{~mm} \times 4 \mathrm{~mm} \times 4 \mathrm{~mm})$ and placed in a six-well plate in a negative-pressure environment for $5 \mathrm{~min}$ to ensure sufficient filling of PRP in the DBMs. 


\section{Scanning electron microscopy (SEM)}

To observe the microstructure of the scaffolds, SEM was used. Neutral glutaraldehyde $(3 \%)$ was used to fix the scaffold and mixture for $4 \mathrm{~h}$, and a series of alcohol gradients (30\%, 50\%, 70\%, 80\%, 90\%, 95\%, 100\%) was then used for dehydration. Each concentration of alcohol was used twice for $15 \mathrm{~min}$. A vacuum freeze dryer was then used for freeze-drying treatment, and SEM images of the microstructures of the DBMs or the DBMs filled with PRP were obtained.

\section{Histological observation of DBMs}

The samples were fixed in $10 \%$ formaldehyde solution for $48 \mathrm{~h}$, rinsed with distilled water and deionized water, and decalcified with ethylenediaminetetraacetic acid (EDTA). The decalcified samples were embedded in paraffin, and 5 - $\mu$-thick sections were obtained from the middle of the sample using a microtome. Samples were then stained with hematoxylin and eosin (H\&E), 4',6-diamidino-2phenylindole (DAPI), and Masson's trichrome. Optical micrographs of the sliced samples were obtained using a microscope.

\section{Immunohistochemical detection of the $\alpha-G a l$ antigen}

As previously described (21), deparaffinized DBMs and PRP-loaded DBM sections were washed three times in PBS and pre-treated enzymatically to enhance immunoreactivity using $0.1 \%$ trypsin for 15 minutes. After washing, samples were soaked in $0.3 \%$ hydrogen peroxide solution at room temperature for 30 minutes. Then, sections were incubated with $3 \%$ normal horse serum (1:200) in PBS to inhibit non-specific binding at room temperature for 1 hour. M86 (1:200; $50 \mu \mathrm{L})$ was used as the primary antibody, and biotinylated horse anti-mouse antibody was used as the secondary antibody. The sections were washed with PBS and developed in DAB solution. Positive results presented as brown deposits on the cell surfaces.

\section{Compressive strength determination}

Five pieces were randomly selected for mechanical measurements on a loading platform using a universal testing machine. First, the long axis of the specimen was kept perpendicular to the loading platform, and the upper and lower planes of the sample were parallel to the loading platform plane, while vertical compression force was applied. The moving speed was set as $5 \mathrm{~mm} \mathrm{~min}^{-1}$, and the loading measurement accuracy was $1 \mathrm{~N}$. The longitudinal deformation of the sample was sensed by an extensometer.
The deformation measurement accuracy was $0.005 \mathrm{~mm}$, and the load-deformation curve and maximum breaking load were recorded by an XY function recorder. The sample had to be kept moist during testing.

\section{Cell proliferation experiment}

DBMs were sterilized and immersed in the culture medium for 24 hours. After being dried, they were transferred to a 24-well plate for later use. Bone marrow mesenchymal stem cells (BMSCs) were used as seed cells. BMSCs were inoculated into samples of each group at a density of 50,000/ well, namely, the blank group (Blank), DBM group (DBMs) and PRP-loaded DBM group (DBMs + PRP). After 1, 4, and 7 days of culture, the cytotoxicity of biomaterials was assessed using the Cell Counting Kit (CCK-8). Specifically, at each time point, after changing the culture medium, a $10 \%$ volume of CCK-8 solution was added and incubated at $37^{\circ} \mathrm{C}$ for two hours. Then, $100 \mu \mathrm{L}$ of the reaction solution was transferred to a new 96-well plate, and the absorbance was measured at $450 \mathrm{~nm}$ by a microplate reader.

\section{Animal experiments}

\section{Implantation}

New Zealand White rabbits $(\mathrm{n}=24)$ were used for scaffold implantation to detect osseointegration in vivo. After anesthesia was induced with intravenous administration of $3 \%$ pentobarbital, an incision was made on the lateral aspect of the left radius. The muscle and subcutaneous tissues were also incised and retracted to expose the radius. A $15-\mathrm{mm}$ bone defect was created, and the periosteum was removed near both ends of the defect to prevent periosteal ossification.

Rabbits were divided into three experimental groups, into which different transplantation materials were implanted ( $\mathrm{n}=8$, each group). The groups included the blank group (Blank), DBM group (DBM group), and PRPloaded DBM group (DP group). Before implantation, a $200-\mu \mathrm{L}$ mixture of thrombin $(0.18 \mathrm{IU})$ and $\mathrm{CaCl}_{2}$ solution $\left(1 \mathrm{~mol} \mathrm{~L}^{-1}\right)$ at a ratio of $1: 1$ was added to the DP group to activate the PRP. The implant was placed in the defect and sutured to the muscle fascia without additional fixation. Hypodermic injection of gentamicin and buprenorphine prevented infection and pain.

\section{Radiographic evaluation post-implantation}

To qualitatively assess graft stability and bridging between the defect and graft, radiographs were obtained of the limbs 

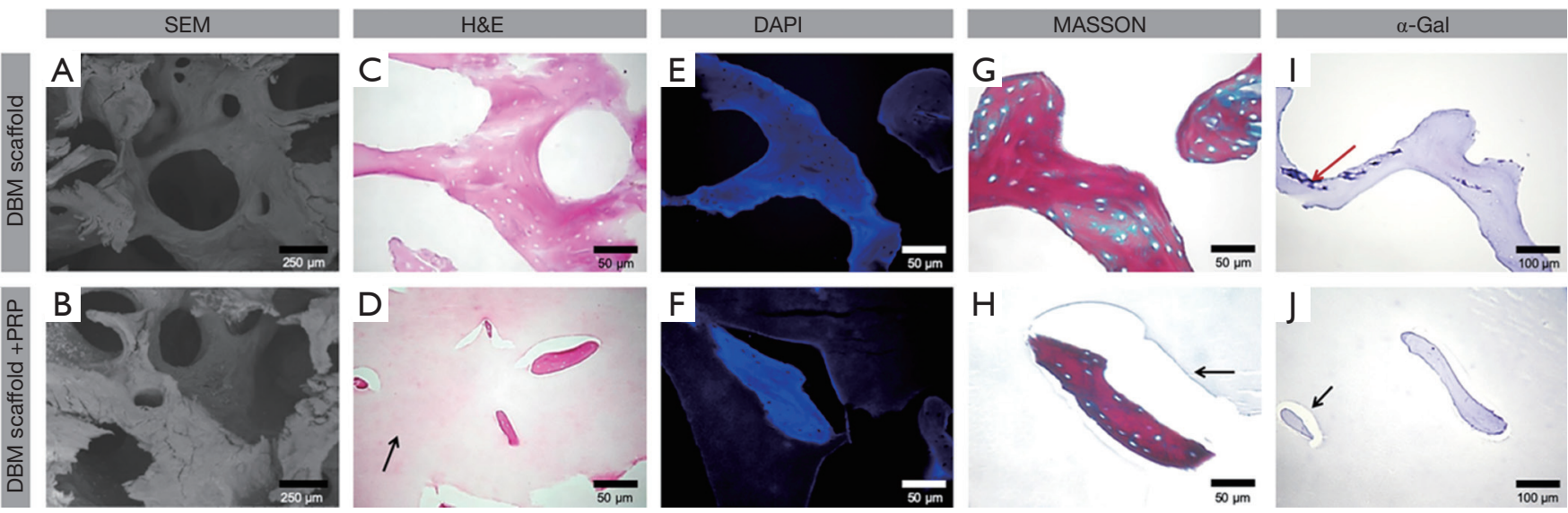

Figure 1 Structural, mechanical and immunogenic properties of DBMs and PRP-loaded DBMs. SEM of DBMs (A) and PRP-loaded DBMs (B). Hematoxylin and eosin (H\&E)-stained DBMs (C) and PRP-loaded DBMs (D). DAPI-stained DBMs (E) and PRP-loaded DBMs (F). Masson-stained DBMs (G) and PRP-loaded DBMs (H). $\alpha$-Gal-stained DBMs (I) and PRP-loaded DBMs (J). The black arrows point to PRP, and the red arrow points to calcification. DBM, decellularized bone matrix; PRP, platelet-rich plasma; SEM, scanning electron microscopy.

under ketamine anesthesia immediately after surgery and at 4,8 , and 12 weeks after surgery.

\section{Histological analysis}

Animals were sacrificed by air embolization at 6 and 12 weeks. The radius was immersed in $4 \%$ polyformaldehyde, decalcified with 12\% EDTA (ethylenediaminetetraacetic acid), and embedded in paraffin wax. Sagittal sections (6 $\mathrm{mm}$ thick) were prepared and stained with Masson's trichrome, H\&E and type I collagen (Col I) for histological assessment. Typical images were obtained using an optical microscope, and digital analysis was performed using Image J software (National Institutes of Health). The areas of new bone, connective tissue, and DBMs within the total area of bone defects were calculated.

\section{Statistical analysis}

The above data are presented as means \pm standard deviations. Data analysis was performed using the SPSS 15.0 software (SPSS Inc., Chicago, IL, USA). The differences were considered significant when $\mathrm{P}$ values were $<0.05$ and highly significant when $\mathrm{P}<0.01$ and $\mathrm{P}<0.001$.

\section{Results}

\section{Characteristics of the DBMs and PRP-loaded DBMs}

The microstructure and immunogenicity of the DBMs and the PRP-loaded DBMs are shown in Figure 1. The SEM images revealed that DBMs and PRP-loaded DBMs exhibited similar matrix morphology (Figure 1A,B). H\&E sections showed that the scaffold itself was stained. There was a circular blank area on the scaffold beam, which may be due to elution of the cells (Figure 1C). PRP is evenly distributed throughout the pores of the scaffold (Figure 1D). DAPI staining showed that the scaffold itself was bluestained (Figure 1E), and no residual nuclei were observed (Figure $1 F$ ), which proved that the scaffold was successfully decellularized. Masson trichrome staining showed a small amount of collagen fibers in the scaffold (Figure 1G,H). No $\alpha$-Gal antigen-positive expression was observed surrounding the DBMs and PRP-loaded DBMs (Figure 11,7). These findings proved that the cancellous bone underwent acellularization and that the $\alpha-\mathrm{Gal}$ antigen was removed effectively. The scaffold was less immunogenic.

Mechanical strength is a critical factor in bioactive scaffolds used for bone regeneration. In the present study, the maximum compression force of DBMs was 295.11 $\mathrm{N}$, compression elastic modulus was $0.003 \mathrm{~N} \mathrm{~mm}^{-2}$, and compressive strength was $14.440 \mathrm{~N} \mathrm{~mm}^{-2}$, which were all close to the parameters of natural bone (22) (Table 1).

\section{Growth factor concentration of PRP}

The PDGF level in PRP was detected by ELISA as $712,100.28 \pm 4,172.78 \mathrm{pg} \mathrm{mL}^{-1}$. The level of bFGF in PRP was $85.44 \pm 37.17 \mathrm{pg} \mathrm{mL}^{-1}$, level of IGF-1 was $198.89 \pm 47.84 \mathrm{pg} \mathrm{mL}^{-1}$, 
Table 1 Biomechanics of DBMs and unprocessed cancellous bone

\begin{tabular}{lccc}
\hline Biomechanics & DBMs & Cancellous bone & P value \\
\hline Maximum compression force & $295.11 \mathrm{~N}$ & $304.01 \mathrm{~N}$ & $>0.05$ \\
Compressive elastic modulus & $0.003 \mathrm{~N} \mathrm{~mm}^{-2}$ & $0.016 \mathrm{~N} \mathrm{~mm}^{-2}$ & $>0.05$ \\
Compressive strength & $14.440 \mathrm{~N} \mathrm{~mm}^{-2}$ & $15.137 \mathrm{~N} \mathrm{~mm}^{-2}$ & $<0.05$ \\
\hline
\end{tabular}

DBM, decellularized bone matrix.

and VEGF level was $89.43 \pm 68.05 \mathrm{pg} \mathrm{mL}^{-1}$.

\section{Cell proliferation experiment}

BMSCs were implanted in each group for 1, 4, and 7 days, and the cytotoxicity of DBMs was evaluated by CCK8 analysis. It was found that the number of cells in each group differed with the increase in culture time (Figure 2). On the fourth day, the proliferation of the DP group was slightly better than that in the other two groups, but there was no significant difference. The proliferation in the Blank group was similar to that in the DBM group. On the seventh day, there was a significant difference between the DP group and Blank group $(\mathrm{P}<0.05)$ and between the DP group and DBM group $(\mathrm{P}<0.01)$. However, the proliferation in the Blank group was not significantly different from that in the DBM group. This result indicates that the DBMs have no obvious cytotoxicity and will not inhibit the proliferation and activity of BMSCs.

\section{In vivo osseointegration of the bone defect}

\section{Radiographic assessment}

Radiographic analyses of bone regeneration and osseointegration between the implants and host bone at 0,4 , 8, and 12 weeks were performed. As shown in Figure 3A,B,C, the critical-size bone defect was constructed in the middle of the radius. The DBMs were evident on the $\mathrm{X}$-ray images, which confirmed the successful and stable scaffold implantation.

Four weeks after implantation, the area of the defect in the blank group was still visible, and no new bone formation was observed (Figure 3D). In the DBM group, new bone formation was observed at the junction of the scaffold and the stump. However, fusion of the stump with the margin of the scaffold was not observed (Figure $3 E$ ). In the DP group, although fusion between the edge of the scaffold and the host bone was not observed, a large amount of new bone was formed within the scaffold, and active bone regeneration was evident (Figure $3 F$ ).

Eight weeks after implantation, the area of the defect in the blank group showed no remarkable changes (Figure $3 G$ ). The DBM group showed a slight increase in implant site density at 4 weeks, and the scaffold was still not effectively integrated with the host bone at 8 weeks (Figure $3 H$ ). The DP group showed obvious fusion between the edge of the scaffold and the host bone. Furthermore, a large amount of new bone was formed within the scaffold, and cortical bone formation was evident on the surface of the scaffold (Figure 3I).

Twelve weeks after implantation, large defects were still evident in the blank group and the DBM group (Figure 37,K, respectively), without any significant bone connections. The repair of bone defects in those two groups showed no significant differences from that at 8 weeks. The amount of new bone in the scaffolds was reduced, and failed osseointegration was evident. In the DP group (Figure 3L), osseointegration was complete after 8 weeks, and reconstruction of the medullary cavity was observed. These observations were the closest to those of natural bone regeneration.

\section{Histological results}

Histological analysis was used to determine the regeneration at bone defects and the components involved, as well as bone integration at the junction of the scaffold and the host bone. Histological analysis (H\&E staining, Masson's trichrome staining, and Col I staining) of retrieved specimens was performed at 6 and 12 weeks after implantation.

\section{Osseointegration connecting position (Figures 4,5)}

At 6 weeks, the bone marrow cavity at the fracture end of the blank group was closed, and the length of the defect was $12 \mathrm{~mm}$. In the DBM and PRP-loaded groups, new bone was observed creeping over the scaffolds to form a stable structure. The scaffolds were covered with a large number 

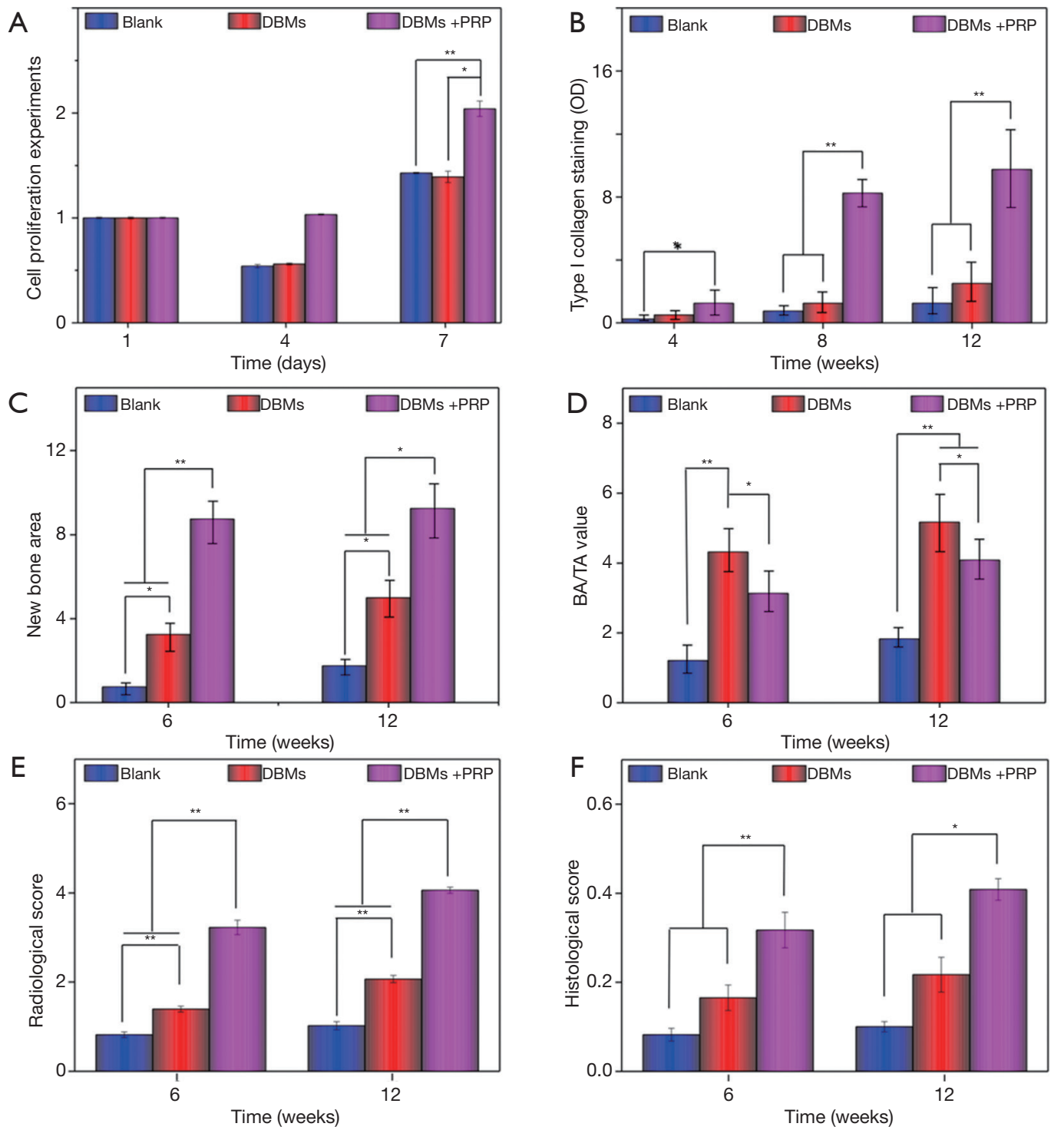

Figure 2 Cell proliferation experiments validate the cytotoxicity of DBMs (A). Type I collagen staining scores (B), new bone area (C), bone area to tissue area (BA/TA) values (D), Radiological scores (E), and histological scores $(\mathrm{F})$. Data are presented as the means \pm SDs, $\mathrm{n}=3$; ${ }^{*} \mathrm{P}<0.05,{ }^{* *} \mathrm{P}<0.01$. DBM, decellularized bone matrix.

of fibrous connective tissues (Figure $4 A, B, C$ ). The blank group showed osteochondrosis at the end of the fracture (Figure 4D, G,7). Braided bone formation was observed at the junction in the DBM group; however, this bone was neither fused with nor embedded in the scaffold (Figure 4E,H). In the DP group, connective tissue proliferation, braided bone formation, and fusion with the scaffold were all observed at the junction from 6 weeks, as well as a small amount of neovascularization and bone regeneration (Figure 4F,I).

The expression of Col I was detected at the defect repair site, indicating that $\mathrm{Col} \mathrm{I}$ appeared at an early stage. The order of Col I expression was: DBMs > blank > DP. Expression at the edge of the graft was lower than that within the graft. These findings show that the addition of PRP effectively prevents the ingrowth of fibers within the scaffolds and provides space for bone tissue regeneration (Figure 47,K,L).

At 12 weeks, the length of the defect showed no change in the blank group (Figure $5 A$ ). In the DBM group, the scaffold surface was covered by a large mass of fibrous 

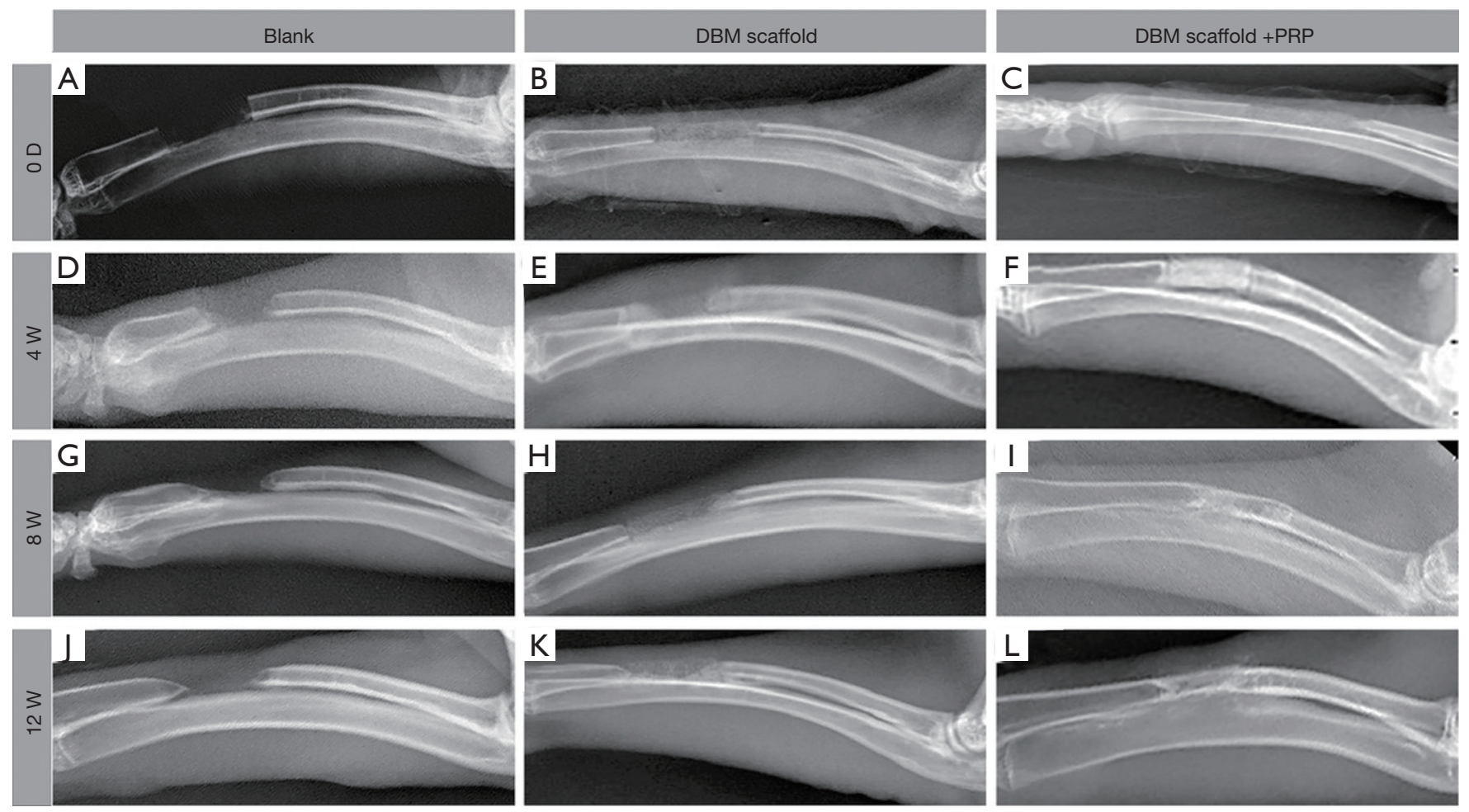

Figure 3 Radiographs of the blank, decellularized bone scaffold matrix (DBM), and platelet-rich plasma (PRP)-loaded groups at different time points after surgery (A,B,C), namely, 4 weeks (D,E,F), 8 weeks (G,H,I), and 12 weeks (J,K,L).

connective tissue, and the original microstructure of the scaffold could not be seen (Figure 5B). In the DP group, the surface of the scaffold was covered by cortical bone, and the scaffold was remodeled to form a structure similar to the natural structure of the radius (Figure 5C). Limited new bone formation was evident at the break, but no woven bone was formed. A significant but small amount of fibroplasia and angiogenesis, the formation of braided bone at the junction, and fusion of the scaffold were all evident in the DP group. Furthermore, neovascularization and new bone formation were evident (Figure $5 E, H, K$ ). Further fusion of the scaffold and stump, trabecular formation, and formation of a large number of new blood vessels and new bone were observed in the DP group (Figure 5F,I,L). The bone integration ability of DBMs in rabbits was improved by the addition of PRP.

\section{New bone formation in the central region of the bone defect}

At 6 weeks, although new tissue formation was evident in the DBM group, this comprised mainly fibrous tissue. A small amount of woven bone was formed at the end of the defect but was neither embedded in nor combined with the scaffold (Figure 6A,B,C). The DP group showed excellent tissue regeneration and separation. In addition, more new bone tissue was formed within the scaffold, which prevented the growth of fibrous tissue. Rich braided, bone-embedded scaffolds were observed at the junction, with only a small gap between the graft and the host bone, and minimal neovascularization (Figure 6D,E,F).

At 12 weeks, the bone defects in the DBM group were poorly healed. The mass of braided bone was slightly increased compared with that at 6 weeks, and a considerable number of collagen fibers were observed within the scaffold. Minimal neovascularization was observed (Figure $7 A, B, C$ ). The bone defects in the DBM group with PRP showed favorable healing, and the degree of bone tissue regeneration and scaffold osseointegration showed further improvement. Fusion between the new bone and scaffold showed extensive neovascularization, and a small amount of cortical bone was observed at the edge of the scaffold (Figure $7 D, E, F$ ). These findings indicate that the DBM group loaded with PRP showed more favorable osseointegration and healing than the DBM group.

Semi-quantitative analysis

To further understand the observations in various groups 

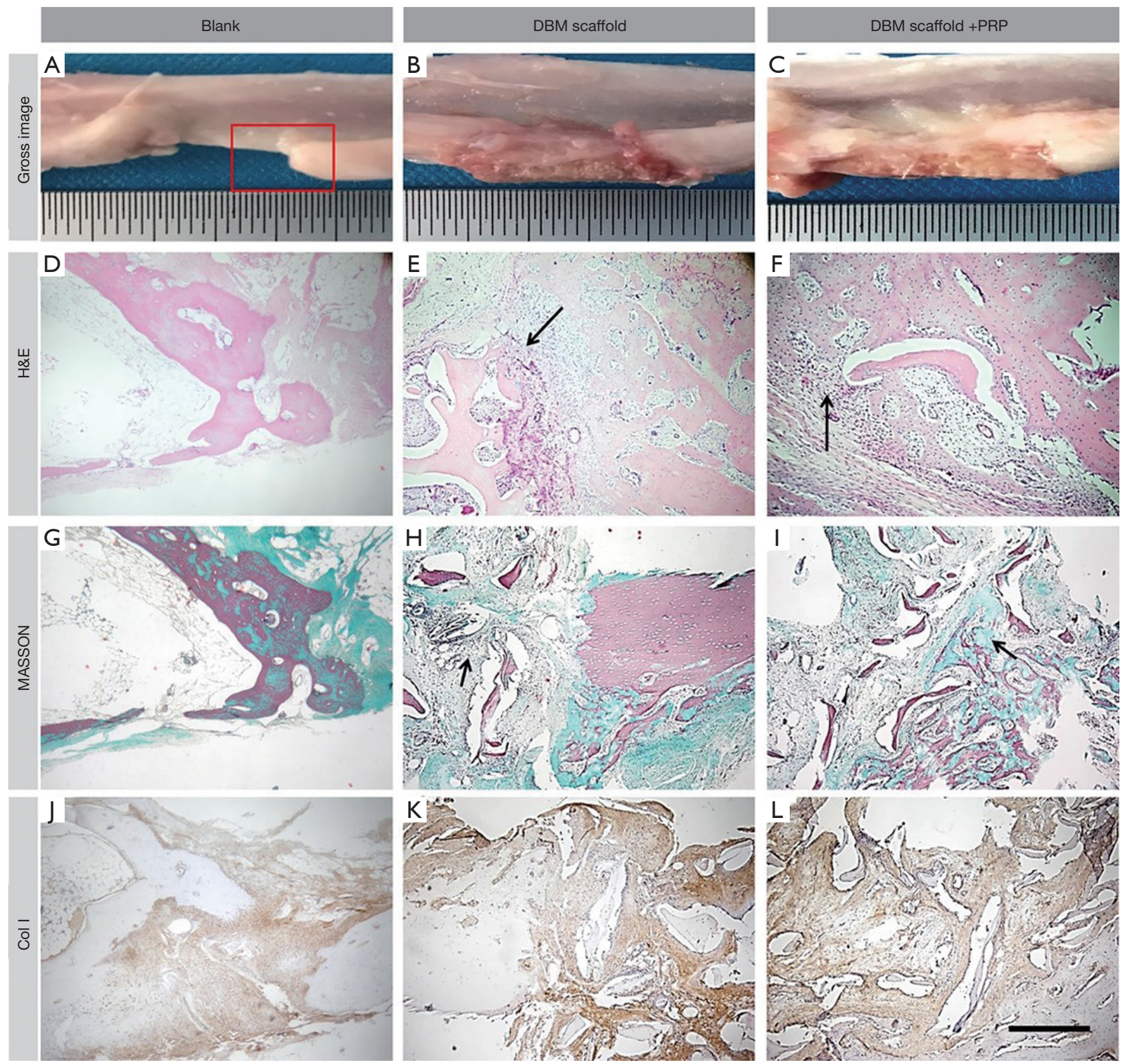

Figure 4 General observation of gross samples (A,B,C) and samples subjected to hematoxylin and eosin (H\&E) staining (D,E,F), Masson's trichrome staining $(\mathrm{G}, \mathrm{H}, \mathrm{I})$, as well as collagen I (Col I) immunohistochemical staining (J,K,L); representative histological images of the graft and host bone junctions 6 weeks after implantation. Black arrows point to new bone tissue. The scale bar indicates $500 \mu \mathrm{m}$.

at different time points, based on the degree of tissue regeneration, we chose the Lane-Sandhu grading standard for imaging and histological grading (23). The absorbance of Col I was measured (Figure 2B), and Image J software was used to measure the new bone area and tissue area ratio (Figure 2C,D). Thus, we found that DBMs were more conducive to promoting bone regeneration. The addition of PRP significantly enhanced the osteogenic ability of DBMs and demonstrated its feasibility in clinical applications (Figure 2). 

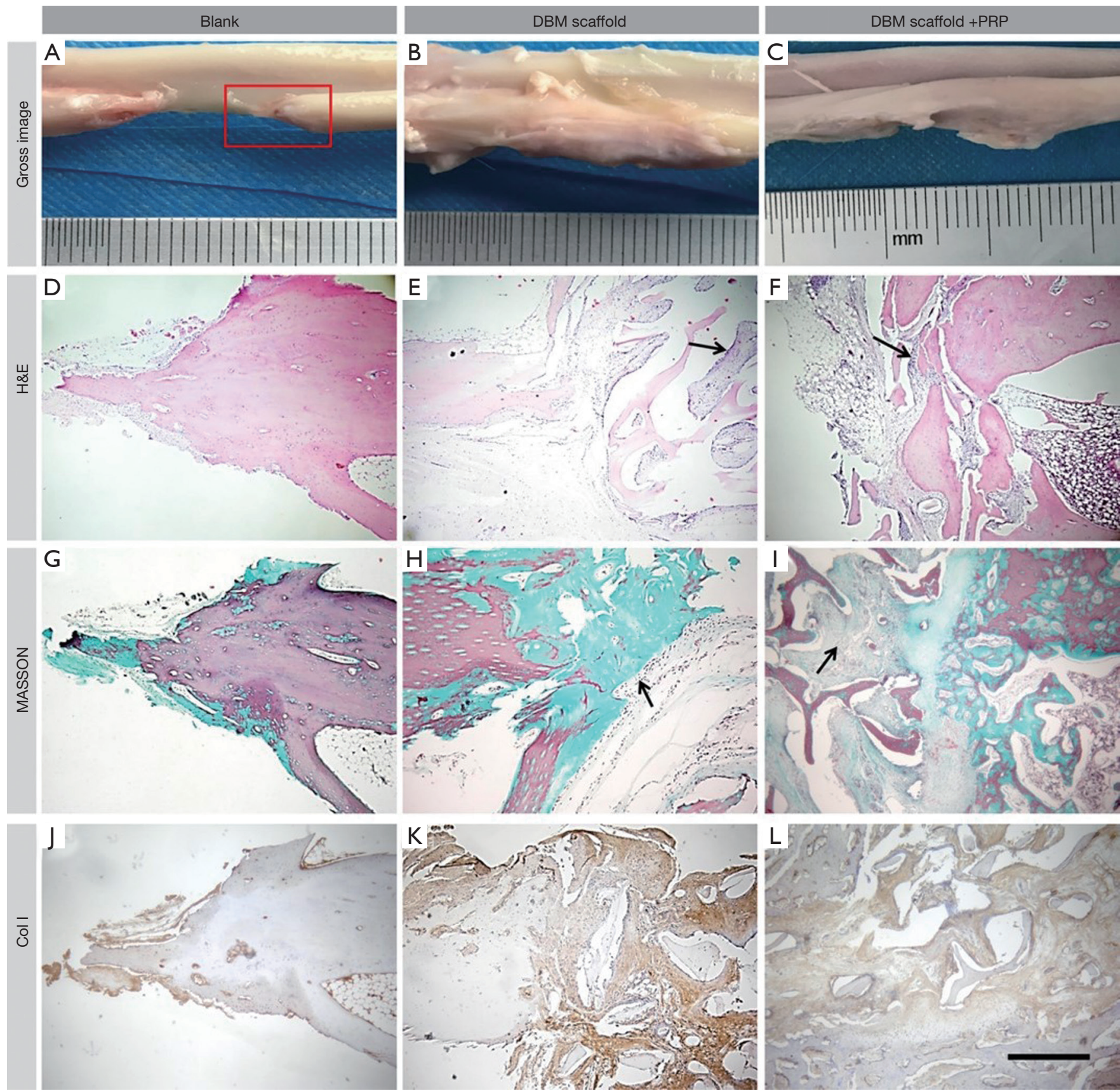

Figure 5 General observation of gross samples (A,B,C) and samples subjected to hematoxylin and eosin (H\&E) staining (D,E,F), Masson's trichrome staining $(\mathrm{G}, \mathrm{H}, \mathrm{I})$, as well as collagen I (Col I) immunohistochemical staining $(\mathrm{J}, \mathrm{K}, \mathrm{L})$; representative histological images of the graft and host bone junctions at 12 weeks after implantation. Black arrows point to new bone tissue. The scale bar indicates $500 \mu \mathrm{m}$.

The imaging scores of the DP group at 4,8 , and 12 weeks $(1.25 \pm 0.5,8.25 \pm 0.5$, and $9.75 \pm 0.957$, respectively) were significantly superior to those of the DBM group $(0.5 \pm 0.577,1.25 \pm 0.5$, and $2.5 \pm 0.577$, respectively $)$ and the blank group $(0.25 \pm 0.5,0.755 \pm 0.957$, and $1.25 \pm 0.5$, respectively) (Figure $2 E$ ). The histological scores of the DP group at 6 and 12 weeks $(7.875 \pm 0.520$ and $9.012 \pm 0.708$, respectively) were significantly superior to those of the DBM group $(2.75 \pm 0.957$ and $4 \pm 0.816$, respectively) and the blank group $(1 \pm 0.816$ and $1.25 \pm 0.957$, respectively) 

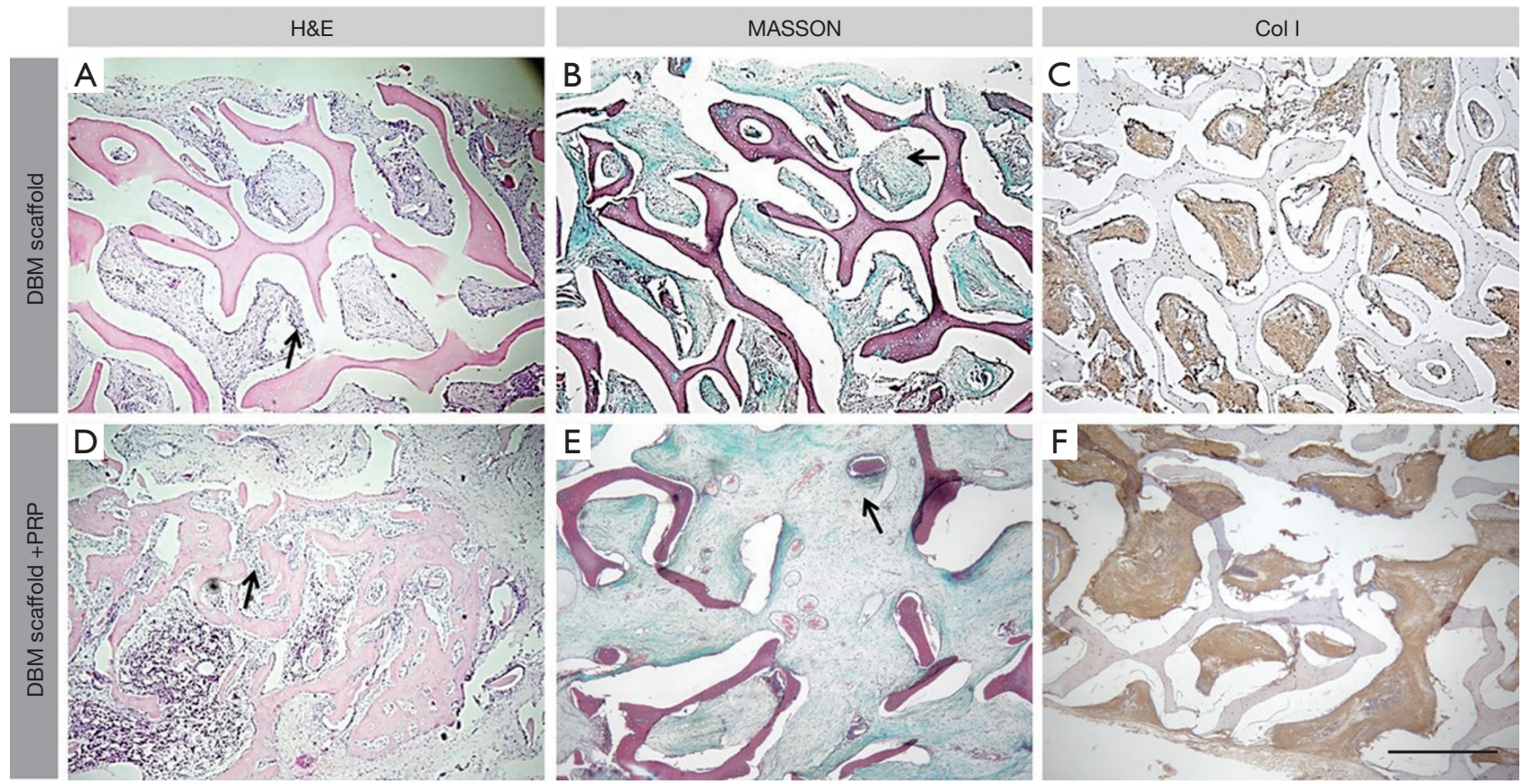

Figure 6 Hematoxylin and eosin $(\mathrm{H} \& \mathrm{E})$ staining $(\mathrm{A}, \mathrm{D})$; Masson's trichrome staining $(\mathrm{B}, \mathrm{E})$; and collagen I (Col I) staining $(\mathrm{C}, \mathrm{F})$ at the central zone of the decellularized bone scaffold matrices (DBMs) and platelet-rich plasma (PRP)-loaded DBM groups 6 weeks after implantation. Black arrows point to new bone tissue. The scale bar indicates $500 \mu \mathrm{m}$.
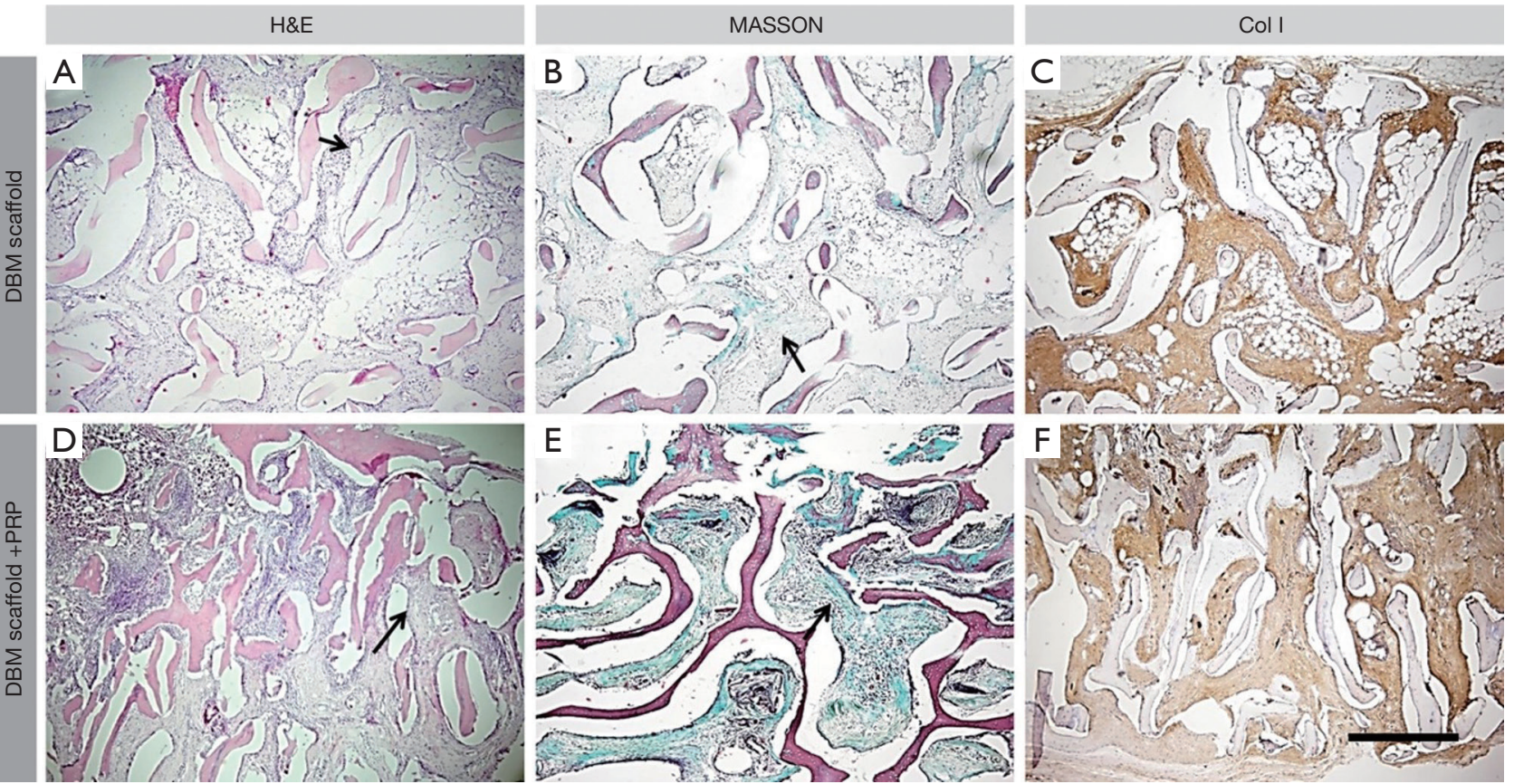

Figure 7 Hematoxylin and eosin (H\&E) staining (A,D); Masson's trichrome staining (B,E); and collagen I (Col I) staining (C,F) at the central zone of DBMs and PRP-loaded DBM groups 12 weeks after implantation. Black arrows point to new bone tissue. The scale bar indicates $500 \mu \mathrm{m}$. DBM, decellularized bone matrix. 
(Figure $2 F$ ). These results indicate that the bone regeneration and bone remodeling abilities of the DP group were higher than those of the other two groups.

\section{Discussion}

To date, autologous bone grafting remains the "gold standard" for clinical bone defect repair. However, its limitations include donor supply, pain, and other complications in the donor area (24). Although several studies have demonstrated the feasibility of bioactive materials as an alternative to tissue repair, only a few successful clinical treatments currently exist for traumatic or pathological bone defects, which require large-scale bone tissue regeneration and repair (25-27).

In this study, we developed an osteoinductive biomaterial comparable to autologous bone, with less likelihood of immune rejection. To completely eliminate immune rejection, a thorough physicochemical treatment of xenogeneic bone tissue was performed to prepare the DBMs. A PRP-loaded DBM composite scaffold was developed to repair the bone defect of the rabbit radius by embedding the PRP into a DBM with a multi-layer structure. The PRP is rich in fibrin and platelets and contains a variety of growth factors and cytokines (28). Among them, PDGF can promote osteoblast proliferation and mitosis of bone marrow stem cells but inhibits osteoblast differentiation. In addition, TGF- $\beta$ stimulates the proliferation and differentiation of osteoblasts. Various concentrations of growth factors were obtained by controlling the platelet concentration of PRP (29).

The PRP-filled scaffold not only provided abundant growth factors but also provided a greater number of bioactive surfaces for cell adhesion. Thus, cell aggregation on the scaffolds was significantly improved (30). Compared with the DBMs, the cell attachment, proliferation, and differentiation of the PRP-loaded DBMs showed significant improvement due to the synergistic effect of fibrin and various growth factors.

Radiographs and histological examination showed that the combination of PRP and DBMs effectively accelerated early wound healing and new bone ingrowth. At 6 weeks after surgery, a good bridge was formed between the fracture end of the humeral defect and the PRP-loaded DBM implant, and a large amount of new bone tissue was formed within the scaffold. The DBM group formed a small amount of new bone tissue.

After 12 weeks, the radial defect implanted with the
PRP-loaded DBMs was almost completely healed. Because the DBMS processing and production removes the immunogenic substances in cancellous bone, as well as the abundant stem cells and osteogenic growth factors normally found in cancellous bone, the defects in the DBMs were not completely repaired. The addition of PRP promoted the growth of new bone tissue at the end of the defect in the radius into the scaffold and significantly promoted the reconstruction of segmental radial defects.

Previous studies have shown that PRP releases a variety of factors after activation by thrombin. These factors have been demonstrated to promote the process of parenchymal cell proliferation and ice-accelerated tissue regeneration. These factors include bFGF, which further induces and maintains bone regeneration by enhancing the proliferation and differentiation of pre-osteoblasts into osteoblasts. The primary effect of PDGF in bone is related to its mitogenic activity. The release of PDGF is beneficial for bone regeneration when applied in wound sites. VEGF enhances neo-vascularization in critical-size bone defects. VEGF can promote the formation of new blood vessels in criticalsize bone defects to transport necessary raw materials and nutrients for bone regeneration (31-35).

However, the efficacy of PRP in osteogenesis remains controversial. The main reasons for this include the following. First, the diversity of the constituents of PRP is the most important factor to consider. Antiplatelet antibody may inhibit the activation of PRP due to an abundance of homologous donor platelets. Therefore, the results may have been mistakenly attributed to PRP. Second, a standardized method of PRP preparation has not yet been established. The platelet concentration of PRP is usually 3-20 times that of whole blood. Growth factors and platelet concentrations depend on the method of manufacture and the type of blood. The concentration of growth factors released by PRP from different species differs widely (36). Low growth factor concentrations in PRP may be ineffective for bone formation, while elevated growth factor concentrations in PRP inhibit osteogenic differentiation. Third, it is difficult to analyze the effects of PRP on osteogenesis. Different species may require different concentrations of PRP to promote bone formation. Thus, the proper range of platelet concentrations for PRP in clinical use remains to be elucidated.

\section{Conclusions}

A functional bone tissue engineering scaffold (DBMs) was prepared by physicochemical processing of xenogeneic 
cancellous bone. Compared with autologous and allogeneic bone grafts, the source of raw materials for xenogeneic cancellous bone was greater. DBMs fabricated by our team were demonstrated to possess certain osteoinductive abilities. Moreover, the addition of autologously derived PRP improved cell adhesion and the capacity of DBMs for osseointegration. To our knowledge, this is the first time that PRP and DBMs have been used in combination to treat critical-size radial defects. Furthermore, PRP improved bone regeneration and integration of DBMs in critical-size defect models of the radial diaphysis in rabbits. The present study may motivate other researchers to further develop xenogeneic cancellous bone and thereby develop new options for the clinical treatment of large bone defects.

\section{Acknowledgments}

Funding: This study was supported by the National Natural Science Foundation of China (Grant No. 81171681, 81701811, 81671804, and 81772456); Scientific Development Program of Jilin Province (Grant No. 20190304123YY, 20180623050TC, and 20180201041SF); Program of Jilin Provincial Health Department (Grant No. 2019SCZT001, 2019SCZT014 and 2019SRCJ001); Cultivation Program from the Second Hospital of Jilin University for National Natural Science Foundation (Grant No. KYPY2018-01); Youth Talents Promotion Project of Jilin Province (Grant No. 192004); Horizontal Scientific Research Project of Jilin University (Grant No. 3R217C923429 and 3R219A693429) and Graduate Innovation Fund of Jilin University (No. 101832018C073).

\section{Footnote}

Conflicts of Interest: HL serves as an unpaid editorial board member of Annals of Translational Medicine from Oct 2019 to Sep 2020. The other authors have no conflicts of interest to declare.

Ethical Statement: The authors are accountable for all aspects of the work in ensuring that questions related to the accuracy or integrity of any part of the work are appropriately investigated and resolved. Institutional Review Board of Jilin University has approved this study (IRB No. 2019132).

Open Access Statement: This is an Open Access article distributed in accordance with the Creative Commons
Attribution-NonCommercial-NoDerivs 4.0 International License (CC BY-NC-ND 4.0), which permits the noncommercial replication and distribution of the article with the strict proviso that no changes or edits are made and the original work is properly cited (including links to both the formal publication through the relevant DOI and the license). See: https://creativecommons.org/licenses/by-nc-nd/4.0/.

\section{References}

1. Niikura T, Lee SY, Sakai Y, et al. Causative factors of fracture nonunion: the proportions of mechanical, biological, patient-dependent, and patient-independent factors. J Orthop Sci 2014;19:120-4.

2. Wang J, Yin Q, Gu S, et al. Induced membrane technique in the treatment of infectious bone defect: A clinical analysis. Orthop Traumatol Surg Res 2019;105:535-9.

3. Lei PF, Hu RY, Hu YH. Bone Defects in Revision Total Knee Arthroplasty and Management. Orthop Surg 2019;11:15-24.

4. Campana V, Milano G, Pagano E, et al. Bone substitutes in orthopaedic surgery: from basic science to clinical practice. J Mater Sci Mater Med 2014;25:2445-61.

5. Fillingham Y, Jacobs J. Bone grafts and their substitutes. Bone Joint J 2016;98-B:6-9.

6. Egol KA, Nauth A, Lee M, et al. Bone Grafting: Sourcing, Timing, Strategies, and Alternatives. J Orthop Trauma 2015;29 Suppl 12:S10-4.

7. Wang ZH, Wang CY, Li C, et al. Analysis of factors influencing bone ingrowth into three-dimensional printed porous metal scaffolds: A review. Journal of Alloys and Compounds 2017;717:271-85.

8. Mauffrey C, Barlow BT, Smith W. Management of segmental bone defects. J Am Acad Orthop Surg 2015;23:143-53.

9. Wu D, Mao F, Yuan B, et al. Minimally Invasive Percutaneous Plate Osteosynthesis (MIPPO) Combined with Onionskin-Like Autologous Bone Grafting: A New Technique for Treatment of Tibial Nonunion. Med Sci Monit 2019;25:5997-6006.

10. Baldwin P, Li DJ, Auston DA, et al. Autograft, Allograft, and Bone Graft Substitutes: Clinical Evidence and Indications for Use in the Setting of Orthopaedic Trauma Surgery. J Orthop Trauma 2019;33:203-13.

11. Ratnayake JTB, Gould ML, Shavandi A, et al. Development and characterization of a xenograft material from New Zealand sourced bovine cancellous bone. J Biomed Mater Res B Appl Biomater 2017;105:1054-62.

12. Bracey DN, Seyler TM, Jinnah AH, et al. A Decellularized 
Porcine Xenograft-Derived Bone Scaffold for Clinical Use as a Bone Graft Substitute: A Critical Evaluation of Processing and Structure. J Funct Biomater 2018. doi: 10.3390/jfb9030045.

13. Li ZJ, Lu CT, Feng ZQ, et al. Antigen-extracted xenogeneic cancellous bone graft with recombinant human bone morphogenetic protein-2 enhances bone regeneration in repair of mandibular defect in rabbits. Kaohsiung J Med Sci 2015;31:18-25.

14. Mautner K, Malanga GA, Smith J, et al. A call for a standard classification system for future biologic research: the rationale for new PRP nomenclature. PM R 2015;7:S53-9.

15. Sánchez M, Delgado D, Pompei O, et al. Treating Severe Knee Osteoarthritis with Combination of Intra-Osseous and Intra-Articular Infiltrations of Platelet-Rich Plasma: An Observational Study. Cartilage 2019;10:245-53.

16. Schneider A, Burr R, Garbis N, et al. Platelet-rich plasma and the shoulder: clinical indications and outcomes. Curr Rev Musculoskelet Med 2018;11:593-7.

17. Teotia AK, Qayoom I, Kumar A. Endogenous PlateletRich Plasma Supplements/Augments Growth Factors Delivered via Porous Collagen-Nanohydroxyapatite Bone Substitute for Enhanced Bone Formation. Acs Biomaterials Science \& Engineering 2019;5:56-69.

18. Kennedy MI, Whitney K, Evans T, et al. Platelet-Rich Plasma and Cartilage Repair. Curr Rev Musculoskelet Med 2018;11:573-82.

19. Qi Y, Niu L, Zhao T, et al. Combining mesenchymal stem cell sheets with platelet-rich plasma gel/calcium phosphate particles: a novel strategy to promote bone regeneration. Stem Cell Res Ther 2015;6:256.

20. Jeong W, Kim YS, Roh TS, et al. The effect of combination therapy on critical-size bone defects using non-activated platelet-rich plasma and adipose-derived stem cells. Childs Nerv Syst 2020;36:145-51.

21. Feng W, Li D, Zang J, et al. Biomechanical comparison of xenogeneic bone material treated with different methods. Xenotransplantation 2017;24:e12343.

22. Liu $X$, Lei $W, W u Z$, et al. Effects of glucocorticoid on $\mathrm{BMD}$, micro-architecture and biomechanics of cancellous and cortical bone mass in OVX rabbits. Med Eng Phys 2012;34:2-8.

23. Lane JM, Sandhu HS. Current approaches to experimental bone grafting. Orthop Clin North Am 1987;18:213-25.

24. Lim ZXH, Rai B, Tan TC, et al. Autologous bone marrow clot as an alternative to autograft for bone defect healing. Bone Joint Res 2019;8:107-17.

25. Tang $W$, Lin D, Yu Y, et al. Bioinspired trimodal macro/ micro/nano-porous scaffolds loading rhBMP-2 for complete regeneration of critical size bone defect. Acta Biomater 2016;32:309-23.

26. Yip I, Ma L, Mattheos N, et al. Defect healing with various bone substitutes. Clin Oral Implants Res 2015;26:606-14.

27. Zhao Y, Wang ZH, Jiang YN, et al. Biomimetic Composite Scaffolds to Manipulate Stem Cells for Aiding Rheumatoid Arthritis Management. Advanced Functional Materials 2019;29.

28. Hesseler MJ, Shyam N. Platelet-rich plasma and its utility in the treatment of acne scars: A systematic review. J Am Acad Dermatol 2019;80:1730-45.

29. Fernandes G, Yang S. Application of platelet-rich plasma with stem cells in bone and periodontal tissue engineering. Bone Res 2016;4:16036.

30. Joo MW, Chung SJ, Shin SH, et al. The Effect of Autologous Platelet-Rich Plasma on Bone Regeneration by Autologous Mesenchymal Stem Cells Loaded onto Allogeneic Cancellous Bone Granules. Cells Tissues Organs 2017;203:327-38.

31. Intini $G$. The use of platelet-rich plasma in bone reconstruction therapy. Biomaterials 2009;30:4956-66.

32. Sohn B, Hwang M, Kim S, et al. Ridge preservation using basic fibroblast growth factor-2 and collagenated biphasic calcium phosphate in beagle dogs. J Periodontal Implant Sci 2017;47:381-7.

33. Liu H, Su H, Wang X, et al. MiR-148a regulates bone marrow mesenchymal stem cells-mediated fracture healing by targeting insulin-like growth factor 1. J Cell Biochem 2018;120:1350-61.

34. Li DQ, Wan QL, Pathak JL, et al. Platelet-derived growth factor BB enhances osteoclast formation and osteoclast precursor cell chemotaxis. J Bone Miner Metab 2017;35:355-65.

35. Huang Y, Pan M, Shu H, et al. Vascular endothelial growth factor enhances tendon-bone healing by activating Yes-associated protein for angiogenesis induction and rotator cuff reconstruction in rats. J Cell Biochem 2020;121:2343-53.

36. Le ADK, Enweze L, DeBaun MR, et al. Platelet-Rich Plasma. Clinics in Sports Medicine 2019;38:17-44.

Cite this article as: Leng Y, Ren G, Cui Y, Peng C, Wang J, Wu D, Liu H. Platelet-rich plasma-enhanced osseointegration of decellularized bone matrix in critical-size radial defects in rabbits. Ann Transl Med 2020;8(5):198. doi: 10.21037/ atm.2020.01.53 